\title{
Outcomes of Macrosurgical Versus Microsurgical Vasovasostomy in Vasectomized Men: a Systematic Review and Meta-analysis
}

\author{
M. Duijn ${ }^{1}$ (D) J. A. van der Zee ${ }^{1} \cdot$ Y. Bachour ${ }^{2}$
}

Accepted: 29 June 2021 / Published online: 20 July 2021

(C) The Author(s) 2021

\begin{abstract}
In order to restore fertility by vasectomy reversal, vasovasostomy (VV) is one of the most chosen options. During this procedure, the vas deferens is anastomosed either by a macro- or microscopical technique. Up to date, it is unknown which of these techniques shows best overall post-procedure patency and pregnancy rates. The purpose of this systematic review and metaanalysis is to reach a consensus on which technique is best for vasovasostomy and thereby better counsel patients and practitioners. A systematic review and meta-analysis on macroscopic and microscopic VV for vasectomy reversal was performed. PubMed, The Cochrane Library, Embase, and Web of Science were systematically searched from inception until 2019. Studies and associated data were evaluated by two reviewers. Primary, data on post-operative patency and pregnancy rates, interval to reversal, and post-operative complications were extracted. Proportions $(95 \% \mathrm{CI})$ and heterogeneity scores $\left(I^{2}\right)$ were calculated, using a random effects model. A total of 8305 patients were included by 25 studies. Descriptive analysis showed higher postoperative patency $(80.5 \%$ vs $91.4 \%)$ and pregnancy rates $(47.7 \%$ vs $73.3 \%)$ after microscopic vasovasostomy. Meta-analysis produced post-operative patency proportions of 0.80 (95\% CI, 0.76-0.84) and 0.88 (95\% CI, 0.83-0.92) after macro- and microscopic VV respectively. Proportions of post-operative pregnancy were 0.43 (95\% CI, 0.35-0.50) after macroscopic VV and 0.47 (95\% CI, 0.31-0.62) after microsurgical VV. Microscopic VV is associated with higher post-operative patency and pregnancy rates compared to macroscopic vasovasostomy. However, further research is needed because of shortage in high methodological quality and variety.
\end{abstract}

Keywords Vasovasostomy $\cdot$ Vasectomy reversal $\cdot$ Microsurgery $\cdot$ Minimally invasive surgical procedure

\section{Introduction}

Vasectomy is a safe and effective form of contraception, and it is estimated that millions of men undergo vasectomy worldwide [1]. In the USA alone, 527,476 vasectomies were

This article is part of the Topical Collection on Surgery

M. Duijn

M.duijn1@amsterdamumc.nl

J. A. van der Zee

J.vanderzee@olvg.nl

Y. Bachour

Y.bachour@amsterdamumc.nl

1 Department of Urology, OLVG, PO Box 95500, 1090 Amsterdam, HM, Netherlands

2 Department of Internal Medicine, Amsterdam UMC- location VUmc, PO Box 7057, 1007 Amsterdam, MB, Netherlands performed in 2015 [2]. During this procedure, the vas deferens is surgically interrupted on both sides with the aim of resulting in permanent infertility. Of all vasectomized men, 3.0-7.4\% request vasectomy reversal because they regret their decision [3-5]. This mainly concerns patients who are sterilized at a young age, couples with a renewed desire for children, and patients with changes in their personal life. The options for vasal reconstruction include vasovasostomy or vasoepididymostomy.

In most patients who desire vasectomy reversal, a vasovasostomy is conducted to reconnect the vas deferens [6]. Within this procedure, a distinction is made between macroscopic and microscopic surgical techniques. The macroscopic method is performed with the naked eye or assisted by a loupe and uses stents or full-thickness sutures. Advantages of this technique are the reduced costs, decreased operative time, and reduced demand for certain operative skills [7]. The microscopic technique is conducted with the support of strong magnification $(16 \times$ to $25 \times)$ and uses full- 
thickness or double-layered sutures [8]. By using these types of magnification, the ends of the vas deferens may be better positioned, which could result in a more accurate alignment of both ends and reduce the chance of postoperative sperm leakage and its associated consequences [8].

Until the mid-1970s, only one method was available: the macroscopic technique, which was the gold standard for vasovasostomy. At that time, the first microsurgical approached vasovasostomy was introduced by Silber (1977), and a new, more precise technique emerged [9-11]. In the past decade, the use of an operation microscope for vasovasostomy has gained popularity, and most expert surgeons use this microsurgical approach. However, some surgeons claim that careful use of macroscopic surgery with reliable materials and experience of the surgeon still results in high postprocedure outcomes.

In recent decades, several studies examining one or both methods have been published. However, an up-to-date review comparing the outcomes for both techniques is missing. Therefore, a review of the currently available data is performed. This study aims to review the literature to obtain a clear picture of the different surgical techniques used for vasovasostomies and to assess which method, macroscopic or microscopic, shows best overall postprocedure outcomes, such as patency and pregnancy rates.

\section{Material and Methods}

\section{Literature Search}

A systematic literature review was performed pertaining to the outcomes of macroscopic vasovasostomy, microscopic vasovasostomy, or both surgical techniques for vasectomy reversal. This systematic review was executed in accordance with the Preferred Reporting Items for Systematic Reviews and Meta-Analysis (PRISMA) statement. The systematic search was performed in PubMed National Library of Medicine, The Cochrane Library, Embase, and Web of Science from inception up to October 2019. The following terms, including synonyms and closely related words, were used: 'vasovasostomy', 'microsurgery', and 'vasectomy reversal'. The full literature search can be found in the Supplementary Information. In addition, a cross-reference search was performed to identify missed relevant articles.

\section{Inclusion and Exclusion Criteria}

Comparative studies between macrosurgical and microsurgical vasovasostomy for vasectomy reversal were considered eligible for inclusion if they reported relevant outcomes such as pregnancy rate, patency rate, postoperative complications, and/or length of interval. All patients had to be first time reversals. Studies on macroscopic or microscopic vasovasostomy alone were also included. The articles were preferably written in English or Dutch. Studies on vasoepididymostomy and robotic vasectomy reversal were excluded. Animal studies, systematic reviews, case report studies, case series, foreign language articles, and articles lacking an abstract were also excluded, as well as studies of patients who underwent vasovasostomy for any reasons other than vasectomy reversal and studies that did not mention any relevant outcomes.

\section{Data Extraction}

The literature search was performed by two reviewers (M. D and Y. B). Any discrepancies between these two authors were resolved by discussion until consensus was reached. First, the title and abstract of the eligible articles were screened. Subsequently, the full text of the selected articles was screened. When the inclusion criteria were met, data extraction was performed using standardized tables. Patient characteristics, type of intervention, postoperative patency and pregnancy rates, mean interval to reversal, the presence of postoperative complications, and time to follow-up were extracted from the included articles. The primary outcomes of interest were postoperative patency and pregnancy rates. Patency is defined as the presence of any motile sperm at postoperative semen analysis without using specific cutoff values. Additionally, pregnancy is defined as a successful natural conception after vasal surgery, regardless of the number, course, and outcome of this pregnancy. Secondary outcomes included postoperative complications (minor or severe), mean interval to reversal (divided into two groups: $\leq 7$ years and $>7$ years), and time to follow-up.

\section{Analysis}

For descriptive analysis, data were subdivided based on the strength of magnification and defined as either macrosurgical or microsurgical. All vasovasostomies that were performed with the use of loupe magnification $(\times 2.5-4)$ or without magnification at all were defined as macrosurgical. In microscopic vasovasostomies, an operating microscope, with strengths ranging from $\times 10$ to $\times 16-25$ and without robotic support, was used. Averages of primary outcomes were calculated to make more reliable and all-encompassing statements when comparing these techniques. These averages were pooled from both retrospective studies and RCTs combined and not only within a certain quality range because heterogeneity scores $\left(\mathrm{I}^{2}\right)$ and results hardly changed when differences in study design were taken into account.

If eligible, studies were included in the meta-analysis. A pooled proportion and the corresponding $95 \%$ confidence intervals were calculated to obtain an overall estimated effect 
size for the association between macro- and microsurgery and postoperative patency and pregnancy. Meta-analysis was performed using a random effects model, and heterogeneity was assessed by the $I^{2}$ statistic. Statistical significance was defined as a 2 -sided $P$ value $<0.05$. All analyses were performed using $\mathrm{R}$ version 3.6.1 ( $\mathrm{R}$ Foundation for Statistical Computing, Vienna, Austria).

\section{Results}

\section{Article Demographics and Patient Characteristics}

In total, 1501 articles were collected through the initial literature search. After removal of duplicates, 839 studies remained. From these articles, 802 were excluded after screening titles and abstracts because of irrelevant subject matter. Of the 37 publications that were eligible for full text screening, twelve were excluded because the relevant outcome parameters were unclear, or they did not mention any of these parameters at all. The remaining 25 studies met the criteria for study inclusion (Fig. 1). These studies were published over an interval of 39 years (1980-2019). The vast majority (22 publications, $88 \%$ ) were performed retrospectively and represented level IIb evidence. The remaining three articles were RCTs (12\%) and represented level Ib evidence according to the Oxford Centre for Evidence-Based Medicine. In the included studies, a total of 8305 patients were included, with an average of 332 per study (range: 12-4010). After loss to follow-up, 5184 patients remained (Table 1). The average age was 38.1 years, and the mean interval to reversal was 7.1 years. A total of 1195 patients underwent macrosurgical vasovasostomy, while the remaining 3989 patients underwent microsurgical vasovasostomy (Table 2). The average year of publication for both modalities was 1999.

\section{Postoperative Patency}

Macroscopic Ten out of 25 included studies (38.5\%) described the macroscopic surgery method for vasovasostomies. Of 671 patients included in these studies, $540(80.5 \%)$ had sperm cells in postoperative semen samples $[7,12,14,17,18,27,32-35]$. Overall, patency ranged from 73.0 to $96.3 \%$ [17, 35]. Moreover, of 246 men with a mean interval to reversal $\leq 7$ years, $189(76.8 \%)$ had patency $[17,27,34,35]$. In 260 patients who reported a mean interval to reversal $>7$ years, $209(80.4 \%)$ had patency [7, $12,18]$. Three studies did not report any mean interval to reversal (Table 2) [14, 32, 33].

Microscopic In nine out of 25 publications (34.6\%), microscopically conducted vasovasostomy was evaluated. A total of $2933(91.4 \%)$ men had patency after surgery, and the overall patency in these studies ranged from 76.5 to $97.9 \%$ [9, 15, 16, 20-24, 31]. Furthermore, 85.2\% (907/ 1064) of the patients with a mean interval after vasectomy $\leq 7$ years had patency, while $78.3 \%$ (216/276) of all patients with a mean interval to reversal $>7$ years showed sperm cells in their ejaculate [15, 20-22, 24]. In four studies, the mean time of interval to reversal was missing (Table 2) [9, 16, 23, 31].

Macroscopic Versus Microscopic Six studies compared macroscopic and microscopic vasovasostomy (Table 2). Lee et al. [13] and Safarinejad et al. [30] compared macrosurgical onelayer and microsurgical two-layer vasovasostomy. The authors of both studies reported patency rates of $90 \%$ and $84 \%$ and $96 \%$ and $82.1 \%$ after macrosurgery and microsurgery, respectively $[13,30]$. Significance was not mentioned by Lee et al. [13]. The difference found by Safarinejad et al. was not statistically significant $(p=0.21)$ [30].

One-layer loupe-assisted $(3-4 \times)$ and one-layer microscopic (10-25×) vasovasostomy were evaluated by Hyun Jee et al. [19] and Hsieh et al. [29] in series of 50 and 63 vasovasostomies, respectively. Hyun Jee et al. showed patency in $72 \%$ after macrosurgery and $96 \%$ after microsurgery [19]. These outcomes were significant $(p=0.021)$. Hsieh et al. reported patency in 25 men $(89 \%)$ and 32 men (91.4\%) after macroscopic and microscopic vasovasostomy, respectively [29]. No significance was found $(p=0.55)$.

Singh et al. [34 compared postoperative patency rates between one-layer vasovasostomy without magnification and one-layer vasovasostomy using a microscope (16-25×). They found patency in $67.6 \%(15 / 23)$ of men who had vasal surgery without magnification and $91.7 \%$ (22/24) after onelayer vasovasostomy by microscopy (16-25×). Significance was not reported [26].

One-layer macrosurgery $(n=300)$ and one- or two-layer microsurgical vasovasostomy $(n=324)$ were evaluated by Yong Lee et al. [28]. After macroscopic surgery, 84\% (252/ $300)$ had patency, while $292(90 \%)$ showed the presence of sperm cells in postoperative semen analysis after microsurgery. These differences were not statistically significant $(P>$ $0.05)$ [28].

Meta-analysis of the fifteen studies reporting postoperative patency rates after macroscopic vasal reconstruction demonstrated a patency proportion of 0.80 (95\% CI, 0.76-0.84) in the random effects model. There was considerable statistical heterogeneity, $I^{2}=65 \%\left(T^{2}=0.0037, p<0.01\right)$ (Fig. 2). In comparison, after analysing the sixteen studies on microsurgical vasovasostomy, the random effects model showed a patency proportion of 0.88 (95\% CI, 0.83-0.92). There was considerable statistical heterogeneity in this model, with $I^{2}=93 \%$ $\left(T^{2}=0.0067, p<0.01\right)$ (Fig. 2). 
Table 1 Studies included in review

\begin{tabular}{|c|c|c|c|c|}
\hline Author (year) & Study design & $\mathrm{N}$, total & Level of evidence & $\mathrm{N}$, after loss to follow-up \\
\hline Kabalin et al. (1991) [12] & Retrospective & 273 & $2 \mathrm{~B}$ & 111 \\
\hline Lee et al. (1980) [13] & RCT & 87 & 1B & 67 \\
\hline Gopi et al. (2007) [14] & Retrospective & 70 & $2 \mathrm{~B}$ & 70 \\
\hline Busato WFS (2009) [15] & Retrospective & 29 & $2 \mathrm{~B}$ & 17 \\
\hline Willscher et al. (1980) [16] & Retrospective & 12 & 2B & 12 \\
\hline C. L. Griffiths (1987) [17] & Retrospective & 20 & 2B & 15 \\
\hline Mason et al. (1997) [18] & Retrospective & 85 & 2B & 79 \\
\hline Hyun Jee et al. (2010) [19] & Retrospective & 56 & 2B & 50 \\
\hline M. Belker et al. (1991) [20] & Retrospective & 1469 & $2 \mathrm{~B}$ & 1224 \\
\hline Aldridge et al. (1985) [21] & Retrospective & 57 & $2 \mathrm{~B}$ & $\begin{array}{l}48 \\
{[52 \text { procedures] }}\end{array}$ \\
\hline Huang et al. (2002) [22] & Retrospective & 70 & $2 \mathrm{~B}$ & 64 \\
\hline M. Fox (1994) [23] & Retrospective & 103 & 2B & 89 \\
\hline Dohle et al. (2005) [24] & Retrospective & 217 & $2 \mathrm{~B}$ & 217 \\
\hline Silber et al. (2005) [25] & Retrospective & 4010 & 2B & 1735 \\
\hline Singh et al. (1995) [26] & RCT & 60 & 1B & 50 \\
\hline Chul Jeon et al. (2017) [27] & Retrospective & 167 & $2 \mathrm{~B}$ & 82 \\
\hline Yong Lee et al. (1986) [28] & Retrospective & 699 & $2 \mathrm{~B}$ & 624 \\
\hline Hsieh et al. (2005) [29] & Retrospective & 74 & $2 \mathrm{~B}$ & 74 \\
\hline Safarinejad et al. (2013) [30] & RCT & 206 & 1B & 196 \\
\hline Cheng et al. (2019) [31] & Retrospective & 51 & $2 \mathrm{~B}$ & 33 \\
\hline Moudi et al. (2016) [32] & Retrospective & 22 & $2 \mathrm{~B}$ & 22 \\
\hline Middleton et al. (1987) [33] & Retrospective & 199 & $2 \mathrm{~B}$ & 73 \\
\hline Bright et al. (2013) [34] & Retrospective & 95 & $2 \mathrm{~B}$ & 95 \\
\hline Denton et al. (1983) [35] & Retrospective & 66 & $2 \mathrm{~B}$ & 54 \\
\hline Feber et al. (1999) [7] & Retrospective & 160 & $2 \mathrm{~B}$ & 83 \\
\hline
\end{tabular}

\section{Postoperative Pregnancy}

Macroscopic The number of pregnancies varied widely across all nine studies that researched the relationship between macrosurgically conducted vasovasostomy and postoperative pregnancy rates, from 26.6 to $63.0 \%$. In total, 255 out of 535 $(47.7 \%)$ women reported one or more pregnancies [7, 12, 14, $17,18,27,32,33,35]$. Strikingly, these studies showed that the length of interval to reversal could possibly influence the chance of pregnancy. Of 144 women whose men had a mean interval to reversal $\leq 7$ years, $89(61.8 \%)$ became pregnant, whereas $37.6 \%$ (85/226) of all women whose husband had a mean interval to reversal $>7$ years managed to conceive $[7$, $12,17,18,27,35]$. One study did not present pregnancy as an outcome, and three studies did not report any useful mean interval to reversal (Table 2) [14, 32-34].

Microscopic Pregnancy rates after microscopic vasovasostomy were documented in eight studies. Of all 2961 women included in these studies, $73.3 \%$ became pregnant after vasal reconstructive surgery of their husbands $[9$, $15,16,20-24]$. Total pregnancy rates ranged from 40.6 to
$91.0 \%[9,22]$. Furthermore, five studies investigated the relationship between the length of interval to reversal and pregnancy rates. Four hundred forty-one (51.3\%) men with a mean interval to reversal $\leq 7$ years managed to become pregnant, compared to $112(42.1 \%)$ pregnancies after a mean interval to reversal $>7$ years $[15,20-22,24]$. Three studies did not report pregnancy rates or a mean interval to reversal (Table 2) $[9,23$, $31]$.

Macroscopic Versus Microscopic Lee et al. [13] and Safarinejad et al. [30] compared macrosurgical one-layer and microscopic two-layer vasovasostomy $[13,30]$. They reported pregnancy rates of $46 \%(19 / 41)$ and $26.7 \%$ (23/94) after macrosurgery and 54\% (14/26) and 28.4\% (29/112) after microsurgery $[13,30]$. Significance was not mentioned by Lee et al. [13]. The differences found by Safarinejad et al. were not significant $(p=0.08)[30]$.

Hyun Jee et al. [19] and Hsieh et al. [29] included a total of 53 women whose husband underwent one-layer loupeassisted $(3-4 \times)$ vasovasostomy. Of these women, $28 \%$ $(7 / 25)$ and $39 \%(11 / 28)$ became pregnant. On the other hand, $40 \%(10 / 25)$ and $43 \%(15 / 35)$ of the women whose men had 


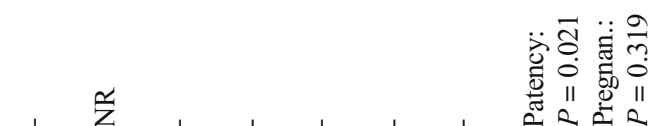

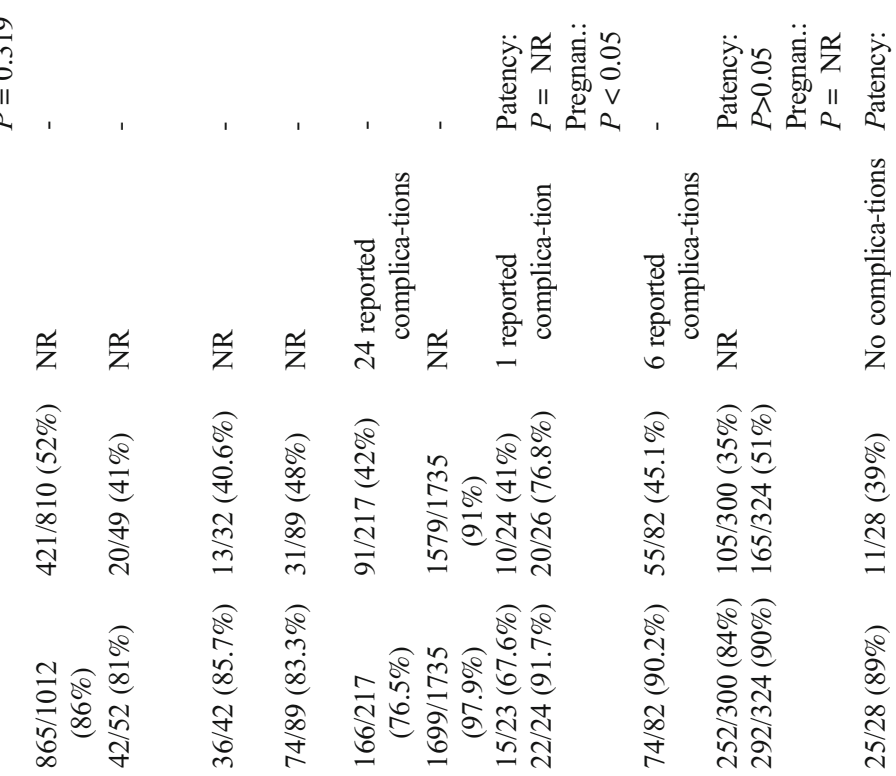

|

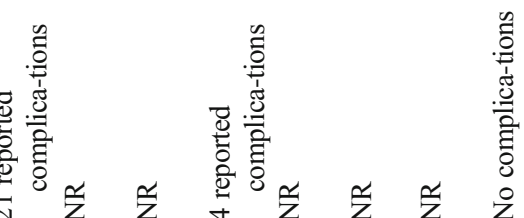

and

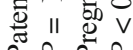

离

\section{ริ)}

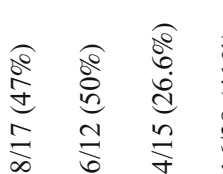

疍

용

휴용

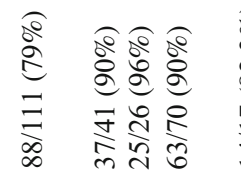

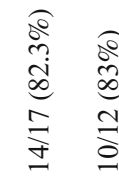

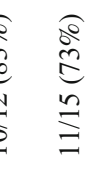

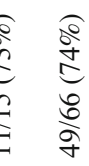

吾:

$\stackrel{n}{n} \stackrel{n}{\infty}$

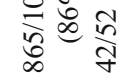

ঙ্ণ

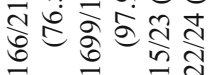

म तु่

त्र

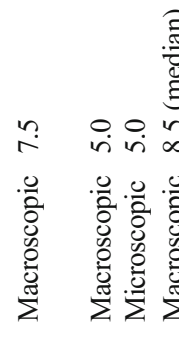

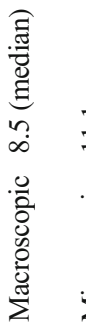

点

ษே.

के

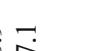

$+\infty \infty$

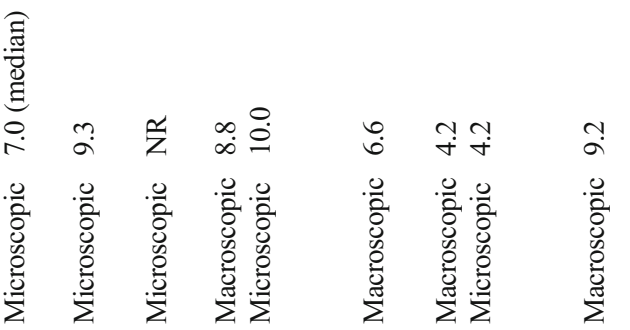

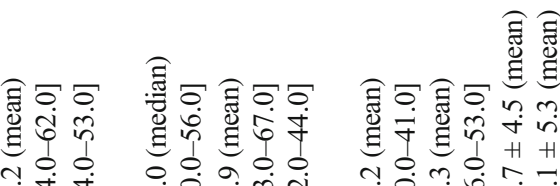

$\stackrel{\infty}{i} \stackrel{\infty}{\sim}$

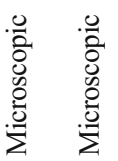

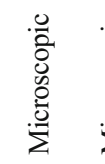

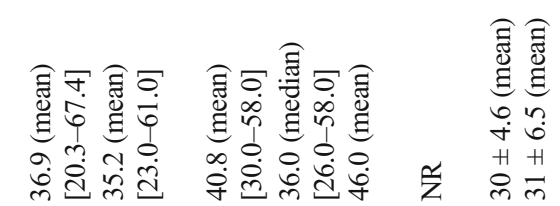

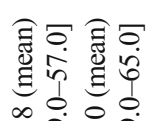

कें 힐.

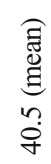

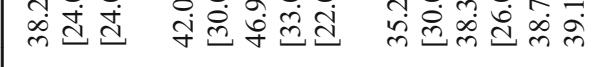

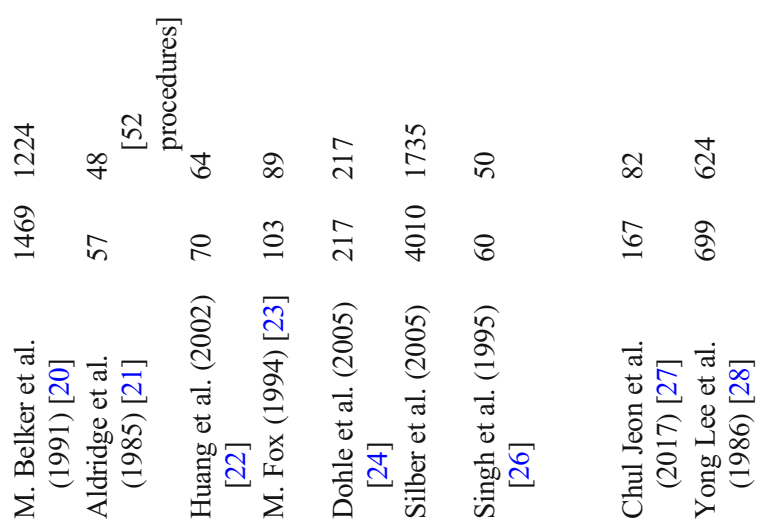




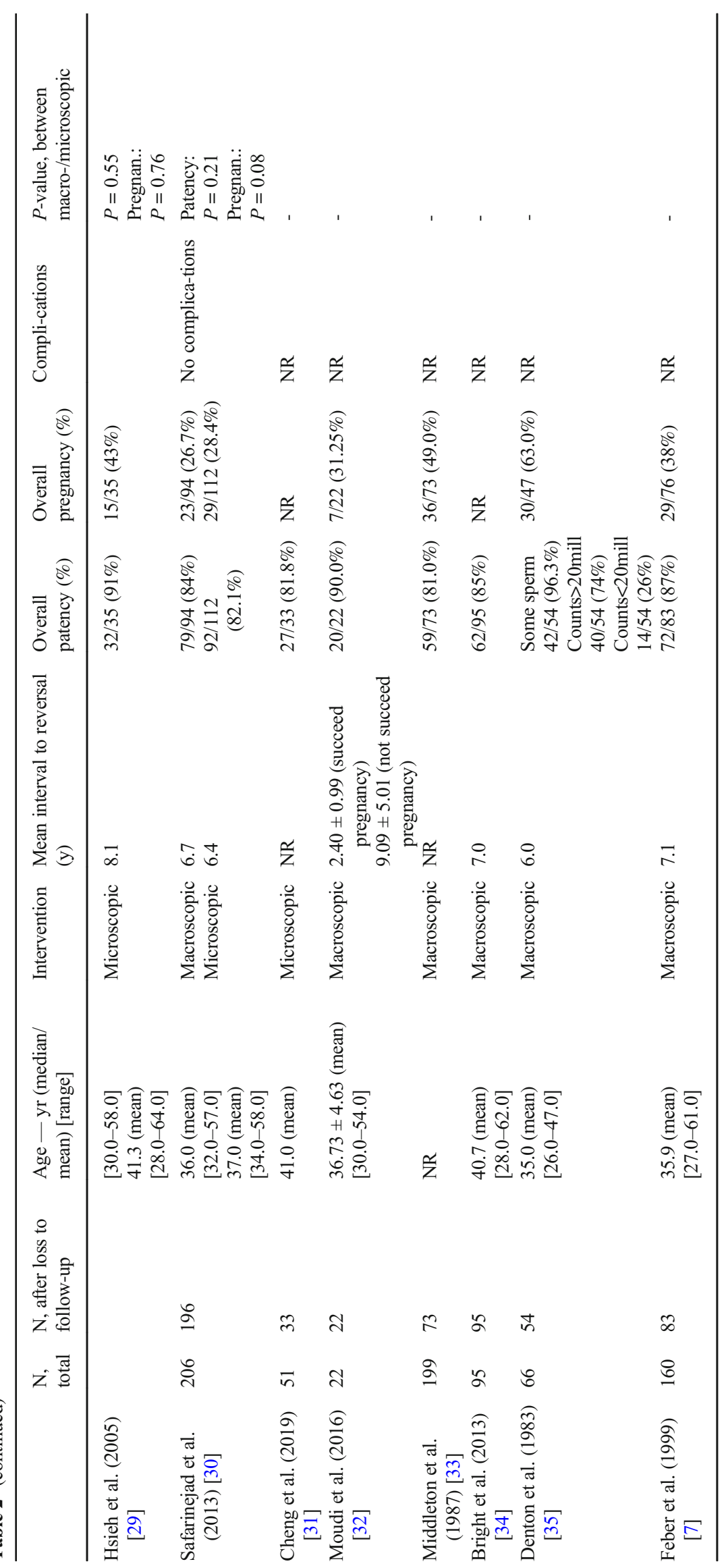


one-layer microscopic $(10-25 \times)$ vasovasostomy reported pregnancy. Corresponding $p$-values showed no significance between groups $(p=0.319$ and $p=0.76)[19,29]$.

Singh et al. [26] did find a significant difference $(p<0.05)$ in pregnancy rates between one-layer vasovasostomy without magnification and one-layer vasovasostomy using a microscope $(16-25 \times)$. Ten $(41 \%)$ women became pregnant after their men had vasal reconstruction without magnification, compared to twenty $(76.8 \%)$ women whose husband underwent microsurgical vasovasostomy [26].

In the study of Yong Lee et al. [28], 105 (35\%) pregnancies were reported after one-layer macrosurgical vasovasostomy and $165(51 \%)$ after one- or two-layer microsurgical vasovasostomy. No $p$-value was reported [28].

A total of fourteen studies on the relationship between postoperative pregnancy rates and macroscopic vasovasostomy were included in the meta-analysis. The random effects model presented a pregnancy proportion of 0.43 (95\% CI, 0.35-0.50), with a statistical heterogeneity of $I^{2}=81 \%\left(T^{2}=0.0138, p<0.01\right)$, which was considerable (Fig. $3)$. Fifteen studies reported postoperative pregnancy rates according to microscopic vasovasostomy. The corresponding pregnancy proportion was 0.47 (95\% CI, 0.31-0.62), and the heterogeneity in this model was considered very high, with $I^{2}=99 \%\left(T^{2}=0.0917, p<0.01\right)$ (Fig. 3).

\section{Complications}

In eight out of 25 publications, the association between surgical technique and postoperative complications was mentioned $[12,15,19,24-27,29,30]$. The overall complication rate after macrosurgical vasovasostomy ranged from 7.3 to $18.9 \%$ (mean: 13.1\%). This concerned mostly minor postoperative

Fig. 1 Flow chart of study selection and inclusion complications, such as haematomas $(n=11)$, wound separation $(n=4)$, and pain $>1$ month $(n=2)$. More severe complications included epididymitis $(n=5)$, wound infections $(n=3)$, and unilateral testicular atrophy $(n=1)[12,27]$.

Furthermore, two studies reported the incidence of postoperative complications after microscopic vasovasostomies [15, 24]. Complications occurred in 28 patients $(17.3 \%)$ and consisted mainly of haematomas, wound infections, and orchitis. Exact numbers were not given. However, none of these complications was specifically related to the microsurgical technique used $[15,24]$. Of the remaining four studies that mentioned complications as an outcome, only one reported a postoperative complication [19, 26, 29, 30]. This complication was not operation related and not further specified or related to an operation technique.

\section{Discussion}

A majority of patients who request vasectomy reversal will eventually undergo vasovasostomy. Since the introduction of the microsurgical technique in 1977 by Silber, different methods for vasovasostomy, macroscopic and microscopic, have been used [9]. To date, it is still not clear which technique is superior and achieves the highest postoperative patency and pregnancy rates. The aim of this review was therefore to systemically review the literature on macroscopic and microscopic vasovasostomy techniques to better counsel patients and practitioners.

We included a total of 8316 patients, of which 5184 remained after loss to follow-up in 25 studies. Most of these publications were retrospective $(n=22)[7,9,12,14-24$, 27-29, 31-35], and three were RCTs (Table 1) [13, 26, 30]. Reviewing the literature showed a mean postoperative

\begin{tabular}{|c|c|}
\hline $\begin{array}{l}\text { The Cochrane library/Web of } \\
\text { science/Central } \\
(\mathrm{N}=444)\end{array}$ & $\begin{array}{l}\text { Embase } \\
(\mathrm{N}=632)\end{array}$ \\
\hline \multirow{2}{*}{$\begin{array}{l}\text { Records after removal of } \\
\text { duplicates } \\
(\mathrm{N}=839)\end{array}$} & \multirow{5}{*}{ 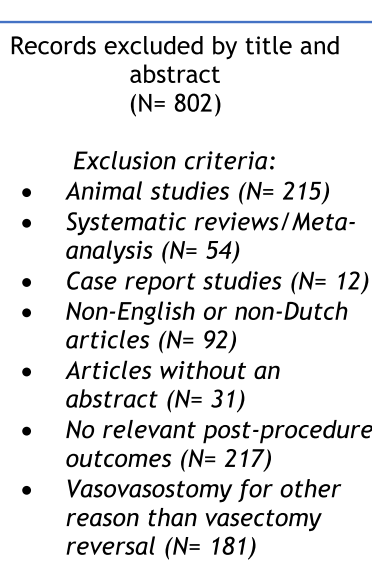 } \\
\hline & \\
\hline $\begin{array}{l}\text { Records after screening title } \\
\text { and abstract } \\
(\mathrm{N}=37)\end{array}$ & \\
\hline $\begin{array}{l}\text { Records included after full } \\
\text { text review } \\
(\mathrm{N}=25)\end{array}$ & \\
\hline & \\
\hline
\end{tabular}


Fig. 2 Meta-analysis of postoperative patency rate comparing macroscopic and microsurgical vasovasostomy; fixed effects and random effects models demonstrated

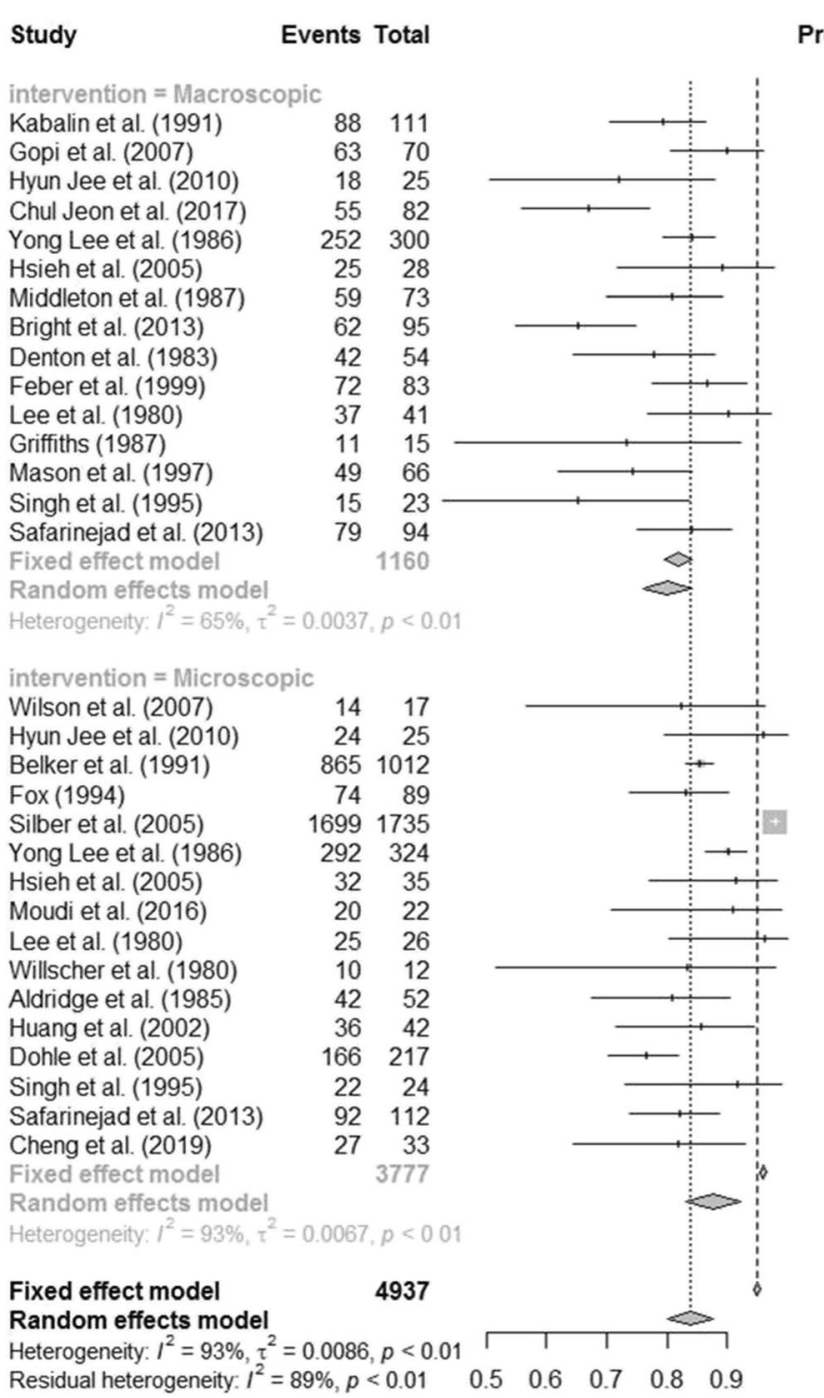

Proportion

$95 \%-\mathrm{Cl}$

Weight Weight

\begin{tabular}{|c|c|c|c|}
\hline .79 & {$[0.71 ; 0.86]$} & $0.6 \%$ & $3.6^{\circ}$ \\
\hline 0.90 & {$[0.80 ; 0.96]$} & $0.7 \%$ & $3.7^{\circ}$ \\
\hline 0.72 & {$[0.51 ; 0.88]$} & $0.1 \%$ & $2.2^{\circ}$ \\
\hline 0.67 & {$[0.56 ; 0.77]$} & $0.3 \%$ & $3.2^{\circ}$ \\
\hline 0.84 & {$[0.79 ; 0.88]$} & $2.0 \%$ & \\
\hline .89 & {$[0.72 ; 0.98]$} & $0.3 \%$ & $3.0^{\circ}$ \\
\hline .81 & {$[0.70 ; 0.89]$} & $0.4 \%$ & $3.4^{\circ}$ \\
\hline 0.65 & {$[0.55 ; 0.75]$} & $0.4 \%$ & 3.3 \\
\hline 0.78 & {$[0.64 ; 0.88]$} & $0.3 \%$ & \\
\hline .87 & {$[0.78 ; 0.93]$} & $0.6 \%$ & $3.6^{\circ}$ \\
\hline 0.90 & {$[0.77 ; 0.97]$} & $0.4 \%$ & 4 \\
\hline 0.73 & {$[0.45 ; 0.92]$} & $0.1 \%$ & \\
\hline 0.74 & {$[0.62 ; 0.84]$} & $0.3 \%$ & 2 \\
\hline 0.65 & {$[0.43 ; 0.84]$} & $0.1 \%$ & 2.0 \\
\hline 0 & {$[0.75 ; 0.91]$} & $0.6 \%$ & \\
\hline 0.8 & {$[0.80 ; 0.84]$} & $7.3 \%$ & \\
\hline & {$[0.76 ; 0.84]$} & & \\
\hline
\end{tabular}

$0.82[0.57 ; 0.96] \quad 0.1 \% \quad 2.1 \%$

$0.96[0.80 ; 1.00] \quad 0.6 \% \quad 3.6 \%$

$0.85[0.83 ; 0.88] \quad 7.3 \% \quad 4.2 \%$

$0.83[0.74 ; 0.90] \quad 0.6 \% \quad 3.6 \%$

$0.98[0.97 ; 0.99] \quad 76.7 \% \quad 4.2 \%$

$0.90[0.86 ; 0.93] \quad 3.3 \% \quad 4.1 \%$

$0.91[0.77 ; 0.98] \quad 0.4 \% \quad 3.3 \%$

$0.91[0.71 ; 0.99] \quad 0.2 \% \quad 2.9 \%$

$0.96[0.80 ; 1.00] \quad 0.6 \% \quad 3.6 \%$

$0.83[0.52 ; 0.98] \quad 0.1 \% \quad 1.8 \%$

$0.81[0.67 ; 0.90] \quad 0.3 \% \quad 3.1 \%$

$0.86[0.71 ; 0.95] \quad 0.3 \% \quad 3.2 \%$

$0.76[0.70 ; 0.82] \quad 1.1 \% \quad 3.9 \%$

$0.92[0.73 ; 0.99] \quad 0.3 \% \quad 3.1 \%$

$0.82[0.74 ; 0.89] \quad 0.7 \% \quad 3.7 \%$

$0.82[0.65 ; 0.93] \quad 0.2 \% \quad 2.8 \%$

$0.96[0.95 ; 0.97] \quad 92.7 \% \quad-\cdots$

$0.95[0.94 ; 0.96] 100.0 \%$ $0.84[0.80 ; 0.88] \quad-\quad 100.0 \%$ patency of $80.5 \%$ and $91.4 \%$ after macroscopic and microscopic vasovasostomy, respectively. The mean incidence of postoperative pregnancy was $47.7 \%$ after macroscopic surgery and $73.3 \%$ after microsurgery. All comparative studies [13, 19, 26, 28, 29], except for Safarinejad et al. [30], supported these higher postprocedure outcomes in favour of the microsurgical technique, without taking into account statistical significance (Table 2). In fact, these results were less consistent when including the interval to reversal. The group of patients with a mean interval to reversal $>7$ years showed higher patency rates after macroscopic vasovasostomy. Additionally, a higher incidence of postoperative pregnancies after macrosurgical reconstruction of the vas deferens in the population of men with a mean obstruction interval $\leq 7$ years was found. Clarification of surgery-related complications in general was not possible due to minimal reported information.

Our meta-analysis of all twenty-two retrospective studies and three RCTs presented similar results as the initial pooled analysis, with statistical evidence in favour of microsurgical vasovasostomy. In both patency ( $0.80 \mathrm{vs} 0.88)$ and pregnancy (0.43 vs 0.47 ), the random effects model demonstrated a higher proportion after microsurgical vasal reconstruction. However, these outcomes are weakened by the high heterogeneity across studies, which in particular affected the analysis by the random effects models of the microscopic technique, with $I^{2}$ values of $93 \%$ and $99 \%$. This variation is mainly caused by differences in population size and surgical methods and to a lesser extent by different follow-up times. Furthermore, bias as a result of the inclusion of the RCTs is unlikely, as the total number of patients per analysis and overall heterogeneity $\left(I^{2}\right)$ did not change dramatically. Because of these unchanged heterogeneous populations, diagnostic accuracy may not have been affected.

Overall, there seems to be a slight advantage to use of the microscope in vasovasostomy. Previous literature has suggested that with the microsurgical technique, a more accurate mucosa-to-mucosa anastomosis between the smaller lumen of the distal vas and the dilated lumen of the proximal vas can be 
Fig. 3 Meta-analysis of postoperative pregnancy rate comparing macroscopic and microsurgical vasovasostomy; fixed effects and random effects models demonstrated

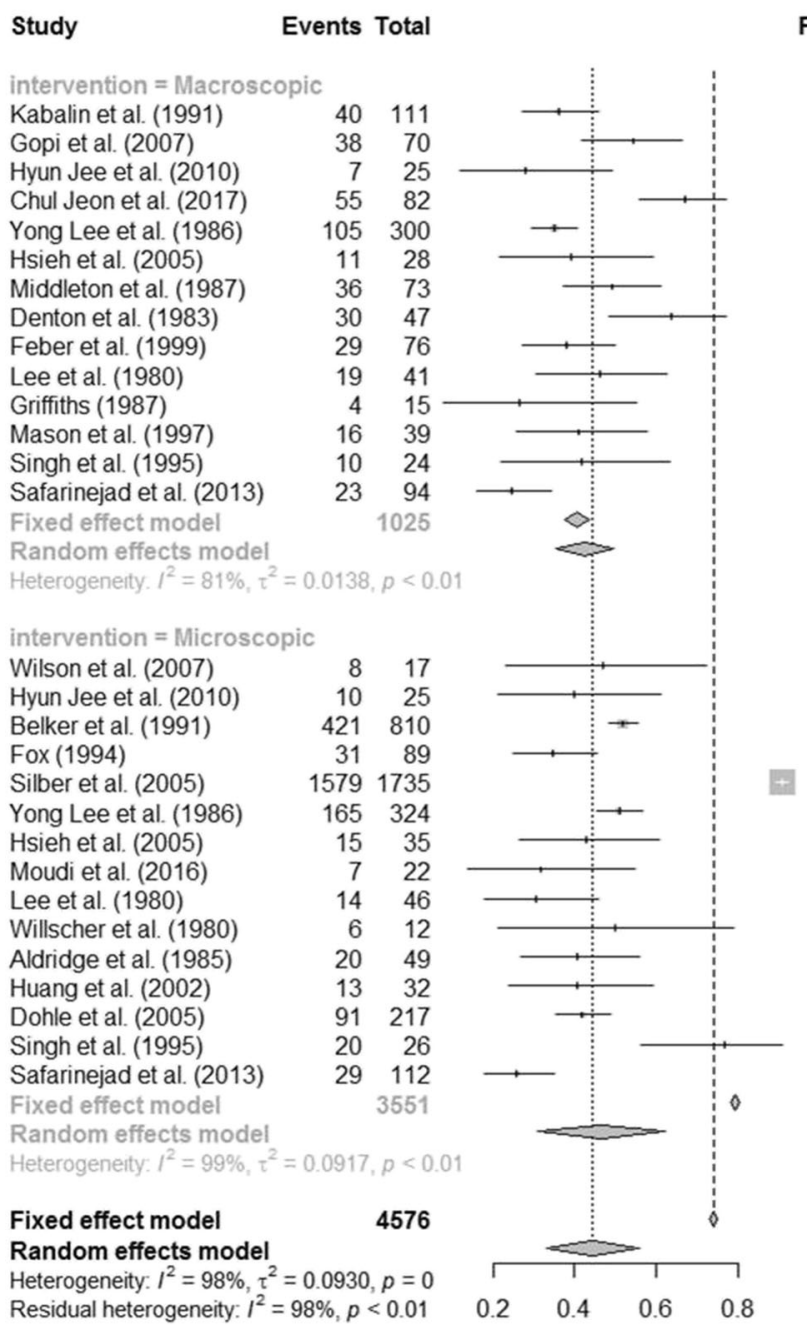

Proportion

$95 \%-\mathrm{Cl}$

Weight Weight

(fixed) (random)

$\begin{array}{lrrr}0.36[0.27 ; 0.46] & 1.4 \% & 3.6 \% \\ 0.54[0.42 ; 0.66] & 0.8 \% & 3.5 \% \\ 0.28[0.12 ; 0.49] & 0.4 \% & 3.4 \% \\ 0.67[0.56 ; 0.77] & 1.1 \% & 3.6 \% \\ 0.35[0.30 ; 0.41] & 4.0 \% & 3.6 \% \\ 0.39[0.22 ; 0.59] & 0.4 \% & 3.3 \% \\ 0.49[0.37 ; 0.61] & 0.9 \% & 3.5 \% \\ 0.64[0.49 ; 0.77] & 0.6 \% & 3.5 \% \\ 0.38[0.27 ; 0.50] & 1.0 \% & 3.5 \% \\ 0.46[0.31 ; 0.63] & 0.5 \% & 3.4 \% \\ 0.27[0.08 ; 0.55] & 0.2 \% & 3.2 \% \\ 0.41[0.26 ; 0.58] & 0.5 \% & 3.4 \% \\ 0.42[0.22 ; 0.63] & 0.3 \% & 3.3 \% \\ 0.24[0.16 ; 0.34] & 1.5 \% & 3.6 \% \\ 0.41[0.38 ; 0.44] & 13.6 \% & - \\ 0.43[0.35 ; 0.50] & - & 48.4 \%\end{array}$

$0.47[0.23 ; 0.72] \quad 0.2 \% \quad 3.2 \%$

$0.40[0.21 ; 0.61] \quad 0.3 \% \quad 3.3 \%$

$0.52[0.48 ; 0.55] \quad 9.7 \% \quad 3.6 \%$

$0.35[0.25 ; 0.46] \quad 1.2 \% \quad 3.6 \%$

$0.91[0.90 ; 0.92] \quad 63.7 \% \quad 3.7 \%$

$0.51[0.45 ; 0.56] \quad 3.9 \% \quad 3.6 \%$

$0.43[0.26 ; 0.61] \quad 0.4 \% \quad 3.4 \%$

$0.32[0.14 ; 0.55] \quad 0.3 \% \quad 3.3 \%$

$0.30[0.18 ; 0.46] \quad 0.7 \% \quad 3.5 \%$

$0.50[0.21 ; 0.79] \quad 0.1 \% \quad 3.0 \%$

$0.41[0.27 ; 0.56] \quad 0.6 \% \quad 3.5 \%$

$0.41[0.24 ; 0.59] \quad 0.4 \% \quad 3.4 \%$

$0.42[0.35 ; 0.49] \quad 2.7 \% \quad 3.6 \%$

$0.77[0.56 ; 0.91] \quad 0.4 \% \quad 3.4 \%$

$0.26[0.18 ; 0.35] \quad 1.8 \% \quad 3.6 \%$

$0.79[0.78 ; 0.80] \quad 86.4 \%$

$0.47[0.31 ; 0.62] \quad-\quad-51.6 \%$

$0.74[0.73 ; 0.75] 100.0 \%$

$0.45[0.33 ; 0.56] \quad-\quad 100.0 \%$ achieved. This precise and watertight anastomosis prevents sperm leakage and thereby the formation of sperm granuloma and strictures [16, 28, 29, 36-38]. Patency of sperm is hereby retained. Approximation of the mucosal edges without microscopic magnification often results in a disparity between both ends due to small anatomical dimensions [29]. Anastomosis is thereby less precise, which in turn affects postoperative patency and pregnancy.

Our findings must be interpreted with caution because of heterogeneity in definitions of postoperative patency and pregnancy. For patency, most studies focused on the presence of any motile sperm cells in the ejaculate at follow-up, while others used minimum sperm counts of 5, 10 or 20 million $/ \mathrm{ml}$. The same applies for pregnancy: the vast majority reported successful, natural, and postoperative conceptions, while some studies reviewed full-term pregnancies or the first postoperative pregnancies only. Because of these inconsistencies, it is difficult to compare all studies.

Another important limitation is that the surgical methods used differed across studies in more than just the strength of magnification. Depending on the surgeon responsible, onelayer sutures, modified one-layer sutures (full thickness), two-layer sutures, or Prolene stents were used for anastomosis. Suture material varied between 6.0, 8.0, 9.0, and 10.0 nylon, and the number of sutures used during vasovasostomy was even less consistent. This makes it hard to determine if the differences in postoperative patency and pregnancy rates were due to the strength of magnification or to the suture techniques and materials used. Furthermore, although scientific research has shown a decrease in female fertility with ageing [39] and physiological variations in sperm density and semen quality among men [40], most studies have not corrected for the age of female partners and the differences in sperm density and semen quality between men. Other limitations of this study were the lack of data on surgeon experience, the retrospective nature of most included studies, different follow-up times, and the lack of comparable studies.

One of the strengths of this study is the clear and reproducible methodology used for performing the systematic review and meta-analysis. 
Furthermore, this systematic review with meta-analysis, executed according to the PRISMA guidelines, is the first to critically examine the introduction of microsurgery for vasovasostomy in comparison with conventional macrosurgery. The ability to pool and analyse different randomized controlled trials and retrospective studies provides an important overview of data and allows statements to be made about the current state of affairs in this area of urological surgery. Urologists, practitioners, and healthcare institutions may now substantiate their preferences and decisions about vasal reconstruction surgery methods based on scientific research.

\section{Conclusions}

Based on a meta-analysis of the best available literature, microsurgically conducted vasovasostomy demonstrated higher postoperative patency and pregnancy rates than macrosurgical techniques. Solely after inclusion of the interval to reversal, some groups seemed to benefit more from macroscopic vasal surgery.

However, definitive conclusions cannot be drawn because of high interstudy heterogeneity owing to discrepancies in surgical methods to anastomose, differences in population sizes, and significant variation in definitions of used endpoints. In view of these data, further research is needed to create the possibility to perform a larger, uniform metaanalysis with a more statistically reliable random effects model.

Supplementary Information The online version contains supplementary material available at https://doi.org/10.1007/s42399-021-01011-1.

Author Contribution Not applicable

Funding Not applicable

Data Availability All data and material will be available.

Code Availability All data and material will be available.

\section{Declarations}

Ethics Approval Not applicable

\section{Consent to Participate Not applicable}

Consent for Publication As corresponding author, I confirm that the manuscript has been read and approved for publication by all the named authors. Consent for publication of an external partner is not applicable.

Conflict of Interest The authors declare no competing interests.
Open Access This article is licensed under a Creative Commons Attribution 4.0 International License, which permits use, sharing, adaptation, distribution and reproduction in any medium or format, as long as you give appropriate credit to the original author(s) and the source, provide a link to the Creative Commons licence, and indicate if changes were made. The images or other third party material in this article are included in the article's Creative Commons licence, unless indicated otherwise in a credit line to the material. If material is not included in the article's Creative Commons licence and your intended use is not permitted by statutory regulation or exceeds the permitted use, you will need to obtain permission directly from the copyright holder. To view a copy of this licence, visit http://creativecommons.org/licenses/by/4.0/.

\section{References}

1. Shattuck D, Perry B, Packer C, Chin QD. A review of 10 years of vasectomy programming and research in low-resource settings. Glob Health Sci Pract. 2016;4(4):647-60.

2. Ostrowski KA, Holt SK, Haynes B, Davies BJ, Fuchs EF, Walsh TJ. Evaluation of vasectomy trends in the United States. Urology. 2018;118:76-9.

3. Potts JM, Pasqualotto FF, Nelson D, Thomas AJ, Agarwal A. Patient characteristics associated with vasectomy reversal. J Urol. 1999;161(6):1835-9.

4. Patel A, Smith R. Vasectomy reversal: a clinical update. Asian J Androl. 2016;18(3):365-71.

5. Valerie U, De Brucker S, De Brucker M, Vloeberghs V, Drakopoulos P, Santos-Ribeiro S, et al. Pregnancy after vasectomy: surgical reversal or assisted reproduction? Hum Reprod. 2018;33(7):1218-27.

6. Schroeder-Printzen I, Diemer T, Weidner W. Vasovasostomy. Urol Int. 2003;70(2):101-7.

7. Feber KM, Ruiz HE. Vasovasostomy: macroscopic approach and retrospective review. Tech Urol. 1999;5(1):8-11.

8. Fenster H, Mcloughlin MG. Vasovasostomy - is the microscope necessary? Urulogy. 1981;18(1):60-4.

9. Silber SJ. Microscopic vasectomy reversal. Fertil Steril. 1977;289(11):1191-202.

10. Ramasamy R, Schlegel PN. Vasectomy and vasectomy reversal: an update. Indian J Urol. 2011;27(1):92-7.

11. Herrel L, Hsiao W. Microsurgical vasovasostomy. Asian J Androl. 2013;15(1):44-8.

12. Kabalin JN, Kessler R. Macroscopic vasovasostomyre-examined. Urology. 1991;38(2):135-8.

13. Lee L, McLoughlin MG. Vasovasostomy: a comparison of macroscopic and microscopic techniques at one institution. Fertil Steril. 1980;33(1):54-5.

14. Gopi SS, Townell NH. Vasectomy reversal: is the microscope really essential? Scott Med J. 2007;52(2):18-20.

15. Busato WFS. Vasectomy reversal: a seven-year experience. Urol Int. 2009;82(2):170-4.

16. Willscher MK, Novicki DE. Simplified technique for microscopic vasovasostomy. Urology. 1980;15(2):147-9.

17. Griffiths CL. Reversal of vasectomy: vasovasostomy. J R Army Med Corps. 1987;133(2):87-8.

18. Mason RG, Connell PG, Bull JC. Reversal of vasectomy using a macroscopic technique: a retrospective study. Ann R Coll Surg Engl. 1997;79(6):420-2.

19. Jee SH, Hong YK. One-layer vasovasostomy: microsurgical versus loupe-assisted. Fertil Steril. 2010;94(6):2308-11.

20. Belker AM, Thomas AJ, Fuchs EF, Konnak JW, Sharlip ID. Results of 1,469 microsurgical vasectomy reversals by the vasovasostomy study group. J Urol. 1991;145(3):505-11. 
21. Aldridge KW, Bueschen AJ, Keith Lloyd L, Burns JR. Microsurgical vasovasostomy for reversal of elective bilateral segmental vasectomy. South Med J. 1985;78(8):967-9.

22. Huang HC, Hsieh ML, Huang ST, Tsui KH, Lai RH, Chang PL. Microsurgical vasectomy reversal: ten-years' experience in a single institute. Chang Gung Med J. 2002;25(7):453-7.

23. Fox M. Vasectomy reversal: microsurgery for best results. Br J Urol. 1994;73(4):449-53.

24. Dohle GR, Smit M. Microsurgical vasectomy reversal: results and predictors of success. Andrologie. 2005;15(2):167-71.

25. Silber SJ, HE Grotjan. Microscopic vasectomy reversal 30 years later: a summary of 4010 cases by the same surgeon. J Androl. 2004;25(6):845-59.

26. Singh I, Kaza RCM. A case in favour of one sided microscopic vasovasostomy - the New Delhi experience. Int Urol Nephrol. 1996;28(1):27-31.

27. Jeon JC, Kwon T, Park S, Park S, Cheon SH, Moon KH. Loupeassisted vasovasostomy using a prolene stent : a simpler vasectomy reversal technique. World J mens health. 2017;35(2):115-9.

28. Lee HY. A 20-year experience with vasovasostomy. J Urol. 1986;136(2):413-5.

29. Hsieh ML, Huang HC, Chen Y, Huang ST, Chang PL. Loupeassisted vs microsurgical technique for modified one-layer vasovasostomy: is the microsurgery really better? BJU Int. 2005;96(6):864-6.

30. Safarinejad MR, Lashkari MH, Asgari SA, Farshi A, Babaei AR. Comparison of macroscopic one-layer over number 1 nylon suture vasovasostomy with the standard two-layer microsurgical procedure. Hum Fertil. 2013;16(3):194-9.
31. Cheng P, Wu W, Chang H, Chung S. The expected outcome of microscope assisted vasovasostomy in Asian people. BJU Int. 2019;123(S3):28-9.

32. Moudi E, Gholam M. Evaluation of successful vasovasostomy with the two-layer macroscopic procedure in Shahid Beheshti Hospital, Babol 2011-2015. International Journal of Advanced Biotechnology and Research. 2016;7(3):2093-8.

33. Middleton RG, Smith JA, Moore MH, Urry RL. A 15-year followup of a nonmicrosurgical technique for vasovasostomy. J Urol. 1987;137(5):886-7.

34. Bright E, Teixiera H, MacDermott JP. Patency rates following a single surgeon's technique for macroscopic vasectomy reversal. BJU Int. 2013;111(3):19-77

35. Denton SE, Bohnert WW, Kurtz CW. Vasectomy reversal technique and results. Ariz Med. 1983;40(1):33-6.

36. Silber SJ. Vasectomy and vasectomy reversal. Fertil Steril. 1978;29(2):125-40.

37. Amelar RD, Dubin L. Vasectomy reversal. J Urol. 1979;121(5): 547-50.

38. Owen ER. Microsurgical vasovasostomy: a reliable vasectomy reversal. ANZ J Surg. 1977;47(3):305-9.

39. Ruman J, Klein J, Sauer MV. Understanding the effect of age on female fertility. Minerva Ginecol. 2003;55(2):117-27.

40. Poland ML, Moghissi KS, Giblin PT, Ager JW, Olson JM. Variation of semen measures within normal men. Fertil Steril. 1985;44(3):396-400.

Publisher's Note Springer Nature remains neutral with regard to jurisdictional claims in published maps and institutional affiliations. 